\title{
Luminescence spectroscopy measurements for skin cancer research
}

\author{
P. Kałużyński ${ }^{1 *}$, Z. Opilski ${ }^{1}$, I. Niedzielska ${ }^{2}$, N. Sitek-Ignac ${ }^{2}$, D. Kogut ${ }^{3}$ \\ ${ }^{I}$ Department of Optoelectronics, Silesian University of Technology, Krzywoustego 2 Str, Gliwice, Poland \\ ${ }^{2}$ Clinic of Cranio-Maxillo-Facial and Dental Surgery, Silesian Medical University, Francuska 20, Katowice, \\ Poland, \\ ${ }^{3}$ Institute of Automatic Control, Silesian University of Technology, Akademicka 2A Str, Gliwice, Poland
}

Received March 12, 2018; accepted March 7, 2008; published March 31, 2018

\begin{abstract}
In this paper we present a simple design of skin cancer optical probe diagnostic system, which uses a non-invasive optical method of detection. This method can be used to image malignancies based on luminescence spectroscopy analysis of given tissues. Through a comparative analysis of red and green peaks in given spectra, the detection of cancerous tissues can be increased. The proposed method and given luminescence spectra show that this system can be used as one of the criteria for early skin cancer diagnosis.
\end{abstract}

Cancerous diseases are one of the most common problems of modern society, being one of the main causes of deaths in developing and highly developed countries. The detection and treatment of tumors are problematic, due to the initially asymptomatic course and rapid development of the pathological process after leaving the period of secrecy. Early detection of dysplastic lesions and tumors in situ is therefore crucial for effective treatment. Due to the latency of the first stages of carcinogenesis, the diagnostic possibilities are very limited, as a result of which the patient gets information about the disease at its advanced stage. This often results in the need to perform radical and mutilating surgical procedures. Often, despite surgical procedures, relapses occur in many patients, which indicates the difficulty in assessing the extent of the cancer process. For example, in a routine study using eye and touch, many subclinical changes go unnoticed. A currently considered most effective way to differentiate pathological processes is histopathological examination. Nevertheless, it is an invasive examination and does not give absolute certainty as to the credibility of the result, because it is possible to obtain an unrepresentative piece of tissue. Therefore, researchers are focusing on the search for non-invasive methods for early cancer diagnosis.

Fluorescence spectroscopy and light-based methods give high hopes for improved detection of, in particular, small tumor foci. The fluorescent spectrum of tissue can be obtained by stimulating it with a suitable laser radiation - which is called laser inducted fluorescence (LIF), or by

${ }^{*}$ E-mail: piotr.kaluzynski@polsl.pl intravenously administering to a patient a small amount of a photosensitizer, which is called photodynamic diagnosis (PDD) [1]. In the first case (LIF), the obtained spectrum is called autofluorescence, natural fluorescence or endogenous fluorescence. In human cells and tissues there are relatively many substances in which the phenomenon of autofluorescence can be induced by ultraviolet light [2]. Amino acids and proteins (tryptophan, collagen fibers, phenylalanine, elastin), coenzymes of metabolic reactions (flavin, FMN, NADH, NADPH), intermediate products of heme synthesis (porphyrins) or lipid metabolism products (lipofuscin) are directly related to this phenomenon [3-5]. Due to fluorescent properties of these compounds, we can observe autofluorescence in the green range of a wavelength spectrum, in response to excitation by UV light [6]. In the second case (PDD), the spectrum obtained is a combination of the autofluorescence spectrum and the fluorescence of the exogenous photosensitizer [7]. It is more beneficial to use the phenomenon of autofluorescence, due to the minimization of interference in the living organism. Naturally occurring porphyrins and their derivatives in human blood may serve as photosensitizers due to their strong absorption of ultraviolet radiation and moreover, they are strongly absorbed and retained in cancer cells. Easy access to narrowband light sources (e.g. lasers, LEDs) also encourages scientists to focus on the use of porphyrins as good photosensitizers, in particular that the absorption maximum is for a $\lambda \approx 405 \mathrm{~nm}[1]$. It is worth noting that the maximum emission of porphyrin fluorescence ranges from 620 to about 670nm, which allows a relatively easy analysis of the maxima of luminescence spectrum signals. In addition, porphyrins are also used in photodynamic therapy, or selective destruction of cancer cells without destroying healthy cells. All the above features were the reason to focus our attention on these chemical compounds in terms of further action and analysis of luminescence spectra. 
This project involved performing blind tests to determine if by spectral analysis we are able to detect cancer changes based on $\mathrm{HpD}$ (hematoporphyrin derivatives) autofluorescence of the samples provided by Cranio-Maxillo-Facial and Dental Surgery Clinic.

Luminescence spectra of given tissues were measured in the UV-Vis range by a laboratory setup shown in Fig. 1, which included an optical spectrometer HR $2000+$ ES (Ocean Optics, USA), fiber optic reflection probe (ArtPhotonics), two bandpass notch filters $(405 \mathrm{~nm})$, and UV laser source (RLDE405M Roithner Lasertechnik). The fiber optic reflection probe contains six optical fibers (to illuminate the sample with UV radiation) placed around one optical fiber, which is used to collect the luminescence signal from the sample.

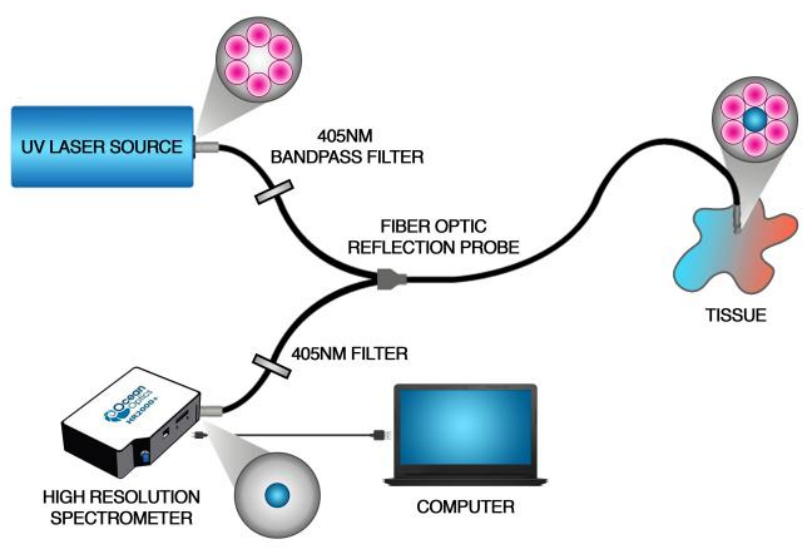

Fig. 1. Scheme of the experimental setup.

Measurements were made for over a dozen series of different types of samples containing both healthy and tumor-altered tissues (Fig. 2). Blind study measurements were made to show differences between the measured samples. After obtaining the luminescence spectra, the results were grouped due to the differentiating features of the spectra obtained, and the blind samples were declassified to compare the validity of the results with the assumptions of the project.

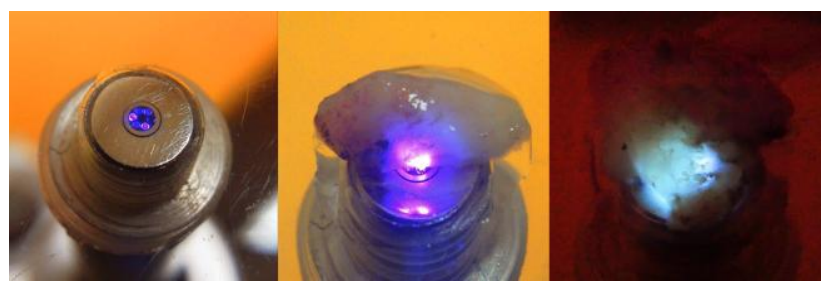

Fig. 2. Photographs of the face of the reflection probe and the measured tissues.

Obtained luminescence spectra (Fig. 3) show specific peaks in the $450 \div 550 \mathrm{~nm}$ range, which is correlated to natural autofluorescence of healthy tissues, which was mentioned in the introduction of this article. The shapes of the spectra are similar to one another. Differences in the intensity of luminescence depend directly on the point at which the sample was illuminated, as well as on the type (gum, tongue) and density of the examined tissue.

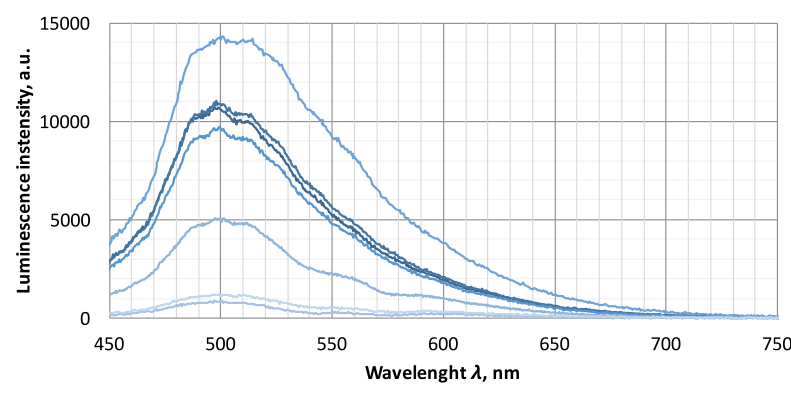

Fig. 3. Obtained healthy tissue luminescence.

The spectra shown in Fig. 4 refer to cancerous tissues. The graph clearly shows the characteristic peaks from the 620 to $650 \mathrm{~nm}$ range, which is caused by the luminescence emission of $\mathrm{HpD}$ accumulated in cancerous tissues. In addition, the level of luminescence of cancerous tissues reaches around 2500 counts at the peak, which is a 5-fold lower signal relative to the level of luminescence of healthy tissues. This phenomenon is most likely due to differences in the tissue content of the reduced and oxidized form of the nicotine nucleotide (NADH and $\mathrm{NAD}^{+}$). Moreover, the blood supply of the diseased tissue and the advantage of given vascularization - venous or arterial, has a significant influence on luminescence levels. Well-vascularized tissues show significant decreases in luminescence in the 420, 540, 580nm bands, which is strictly related to the maximum absorption spectrum of hemoglobin.

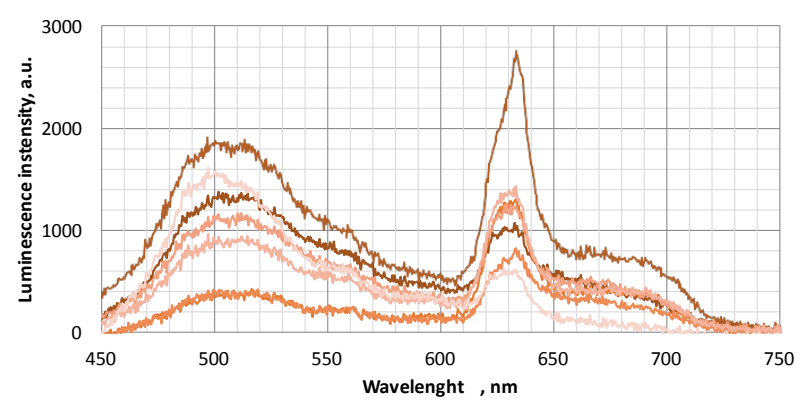

Fig. 4. Obtained cancerous tissue luminescence

In conclusion, series of luminescence spectroscopic measurements were made for healthy and cancerous tissues. The grouping of the obtained results allowed to indicate characteristic features for the obtained spectra for 
healthy and diseased tissues. Based on the HpD luminescence, the differences between the spectra of healthy and cancerous tissues are so characteristic that it is easy to classify whether pathological changes have occurred in the tissue or whether the tissue is healthy. Through a comparative analysis of red and green peaks in given spectra, the detection of cancerous tissues can be increased. The most important features of the presented method are its non-invasiveness, sensitivity and specificity, as well as the speed of acquiring information. The "optical biopsy" method does not require material collection, and the amount of material analyzed is essentially unlimited. The given results clearly show that the proposed method of "optical biopsy" can be used as an equal complement to classic methods (e.g. histopathology) of early detection of cancer.

The work was partially sponsored by the Faculty of Electrical Engineering of the Silesian University of Technology within the grant BKM/534/RE4/2015 and Silesian Medical University in Katowice.

\section{References}

[1] A. Sieroń, W. Stręk, H. Podbielska (Urban\&Partner, 2004).

[2] A. Profio, IEEE J. Quantum Electr. QE-20(12), 1502 (1984), DOI: 10.1109/JQE.1984.1072348.

[3] S. Palmer, J. Biophoton. 10(8), 1062 (2017), DOI: 10.1002/jbio.201600162.

[4] M.A. D'Hallewin, Europ. Urology, 42(5), 417 (2002), DOI: $10.1016 / \mathrm{S} 0302-2838(02) 00402-5$.

[5] M. Monici, Biotechnol. Annual Rev. 11, 227 (2005), DOI: $10.1016 / \mathrm{S} 1387-2656(05) 11007-2$

[6] A.C. Croce, G. Bottiroli, Europ. J. Histochem. 58(4), 2461 (2014), DOI: 10.4081/ejh.2014.2461.

[7] G. Bottiroli, A.C. Croce, Photosciences, lasers and current optical techniques in biology (RSC Books and Database, 2004).

[8] R.A. Drezek, K.V. Sokolov, U. Utzinger, I. Boiko, A. Malpica, M. Follen, R.R. Richards-Kortum, J. Biomed. Opt. 6(4), 1 (2001). DOI: 10.1117/1.1413209. 